\title{
Arterial pulsation on a human patient simulator improved students' pulse assessment
}

\author{
Akihiro Takeuchi $^{1}$, Tomomi Kobayashi ${ }^{1}$, Minoru Hirose ${ }^{2}$, Takashi Masuda ${ }^{3}$, Toshiro Sato ${ }^{1}$, \\ Noriaki Ikeda ${ }^{1}$ \\ ${ }^{1}$ Department of Medical Informatics, School of Allied Health Sciences, Kitasato University, Sagamihara, Japan \\ ${ }^{2}$ Department of Medical Safety Engineering, School of Allied Health Sciences, Kitasato University, Sagamihara, Japan \\ ${ }^{3}$ Department of Rehabilitation, School of Allied Health Sciences, Kitasato University, Sagamihara, Japan \\ Email: take@kitasato-u.ac.jp, ce08714e@st.kitasato-u.ac.jp, hirose@kitasato-u.ac.jp, tak9999@med.kitasato-u.ac.jp, \\ toshiro_sato@jcom.home.ne.jp, ikeda@kitasato-u.ac.jp
}

Received 28 February 2012; revised 17 March 2012; accepted 5 April 2012

\begin{abstract}
Even with basic cardiovascular lectures, undergraduates do not usually experience the reality of palpation and, therefore, cannot integrate their physiological knowledge. We created a pulse training scenario of human patient simulators (HPS) to recognize and assess the normal and arrhythmic pulse of the radial artery. All 25 participants were recruited as volunteers to the study from the School of Allied Health Sciences, Kitasato University. Participants received training in radial palpation of arrhythmias on HPS. The test scenario included 10 arrhythmic pulses combined with normal pulses and weak pulses. The average examination scores significantly improved, from $23.8 \pm 2.8$ of the pretest to $72.9 \pm 3.4$ of the posttest (mean and SE, $\mathrm{N}=25, \mathrm{p}<0.00001$ ). A questionnaire and general written comments for the palpation training were positive. The palpation training improved the participants' assessment of radial pulses.
\end{abstract}

Keywords: Education; Physiology; Human Patient Simulator; Arrhythmic Pulse; Pulse Assessment

\section{INTRODUCTION}

Detailed physical examinations frequently provide important information needed to assess the cardiovascular system. The first step in evaluating patients with palpitations is to determine whether or not their symptoms are actually due to arrhythmias. Palpation of the carotid and radial arteries is simple and often an underutilized lowcost method [1]. Pulse characteristics must be assessed simultaneously and carefully for rate (tachycardic, normal, or bradycardic, or any irregularity), and volume. Irregular pulse can be regularly irregular e.g. bigeminy or irregularly irregular e.g. atria fibrillation [2]. Although arrhythmias are easily assessed with an electrocardiogram
(ECG) and Holter monitoring to determine the type, precise palpation of the arterial pulse helps in diagnosing arrhythmias, even asymptomatic ones, and is also necessary to determine blood flow adequacy. Although it is simple for medical staff to understand the importance of palpation, many undergraduate students do not realize their arterial pulses as cardiovascular events and how they are related to their own physiology. There is likely dissociation between their knowledge and reality. Handson experience of their own normal pulse and pulses of arrhythmias, even with virtual reality devices, should deepen their understanding of cardiovascular physiology.

Development of human patient simulators (HPSs) began in the late 1960s and accelerated in the late 1980s and early 1990s [3]. HPSs are used at medical centers, universities, and colleges in the USA and throughout the world $[3,4]$. HPSs are also used in the education and training of healthcare professionals and undergraduates in scientific inquiry [3,5-7]. They are used to teach basic skills, such as respiratory physiology and cardiovascular hemodynamics, and advanced clinical skills, e.g. management of difficult airways, tension pneumothorax, pulmonary embolism, and shock [3]. However, we could not obtain an HPS scenario that focused on the palpation skill of arterial pulses and arrhythmias.

We created an original simple training scenario of arterial pulses and arrhythmias so that the students can realize the relationships between pulses and cardiovascular hemodynamics. The palpation training was evaluated by pre- and postexamination and a questionnaire. Ethical approval for this study was granted by the Kitasato University Medical Ethics Committee, 6 September 2011, Section B 11-76.

\section{MATERIALS AND METHODS}

\subsection{Participants}

All the participants, aged between 20 and 27 years, were 
recruited as volunteers to the study $(21.5 \pm 0.5$ mean \pm SE; 16 males, 9 females; clinical engineering course 23, occupational therapy course 2) from the School of Allied Health Sciences, Kitasato University. Certain participants had an explicit interest in emergency medicine and had experience of AED (automated external defibrillator) training. All of the participants had been lectured on cardiac physiology and cardiac diseases. This research ethics board approved the study and consent was obtained from all participants.

\subsection{Procedure and Design}

We created a palpation training scenario of HPS to recognize characteristics of pulse sequences related with arrhythmia. The scenario included six arrhythmias, normal sinus rate, bradycardia, tachycardia, atrial fibrillation (AF), ventricular premature contraction (bigeminy), and arrest. They were combined with normal pulse or weak pulse on the radial artery (Figure 1).

Participants were trained on arterial palpation of arrhythmias on HPSs in a mini lecture. They could palpate the pulse as many times as they wanted. The participants were asked to describe their assessment for each pulse examination before the mini lecture (pretest) and again after the mini lecture (posttest). In the examinations, 10 kinds of arrhythmias were sequentially presented by an instructor in each scenario. The ECG monitor was not presented merely to train the palpation techniques. Each participant was requested to palpate the HPS's radial artery and assess and write a diagnosis within 1 minute for each arrhythmia. The response form was not a multiple choice form to avoid guesses as answers. After the examinations, the participant was asked to answer the questionnaire to evaluate the training using a 4-point scale ranging from 'agree strongly' to 'disagree strongly' and to add comments on his or her impressions of the experience.

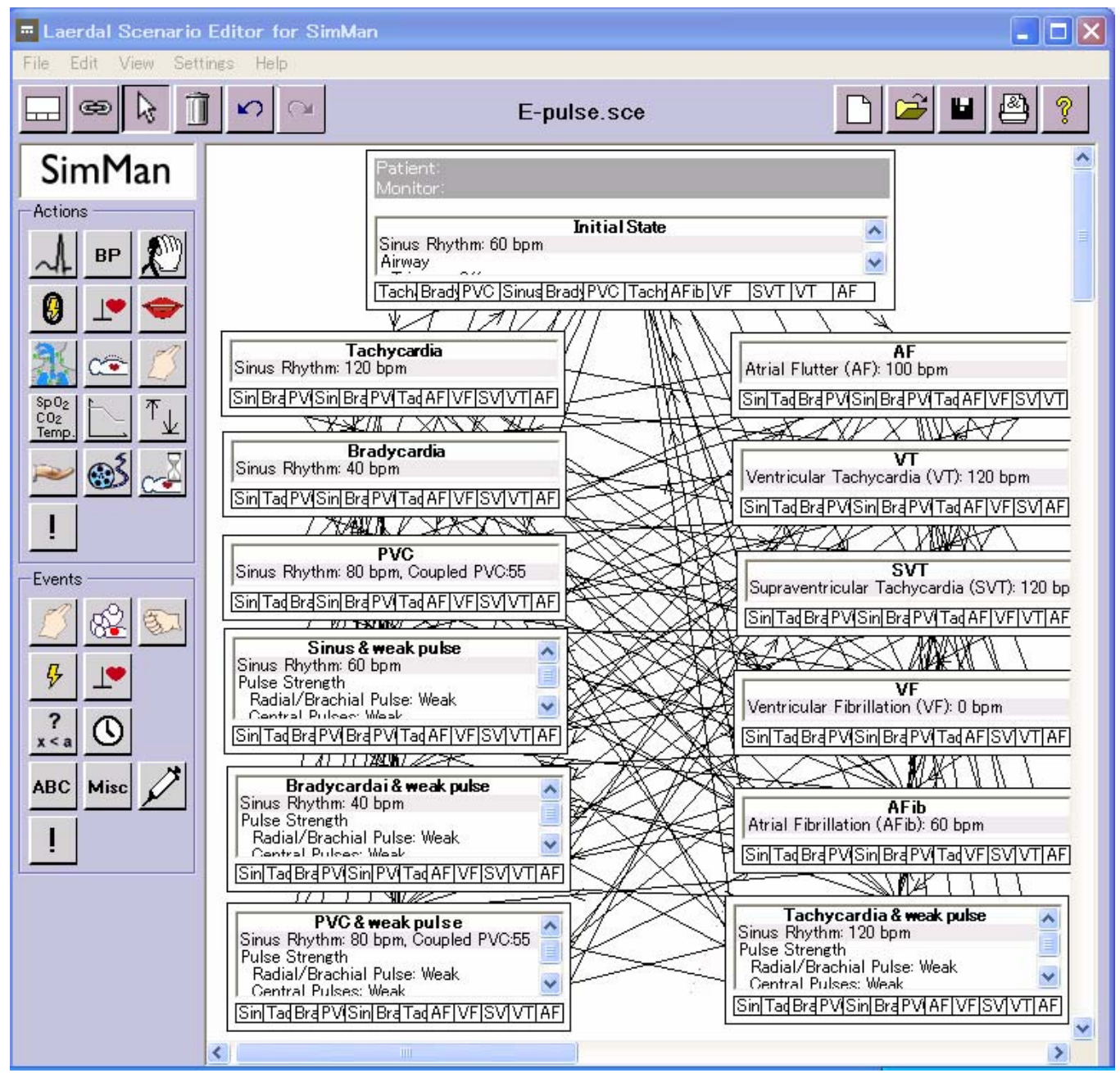

Figure 1. Creating the scenario of the palpitation training on a scenario editor in the HPS. Each box frame in the window includes a setting of pulse rhythm, pulse rate, and pulse strength on HPS. The complicated arrow lines between the frames indicate the next setting. 


\subsection{Statistical Analysis}

Test scores were summarized for each student's pretest and posttest. The scores were summarized using descriptive statistics including means and standard errors (SEs). Comparison of the scores was accomplished using pared $t$-test analysis. The scores were considered significantly different at $p \leq 0.05$.

\section{RESULTS}

The training scenario including 10 arrhythmic pulses combined with normal pulses or weak pulses was manually created with the scenario editor of the HPS.

After the scenario was manually loaded and run, arrhythmia menu was listed in a control window (Figure 2). Requested arrhythmia pulses were presented on radial and cervical artery of the HSP. The mean of test scores was significantly improved from $23.8 \pm 2.8$ of pretest to $72.9 \pm 3.4$ of posttest (mean and SE, $N=25$, p $<0.00001$ ) (Table 1). Questionnaires and general written comments were very positive (e.g. "promotes critical thinking," “practice without risk," etc.) Ninety-six per cent of the students rated the simulator training as "excellent" or "very good".

\section{DISCUSSION}

HPS has various basic functions and a scenario construc- tor/editor to create a new training scenario of specific clinical cases. Many various scenarios were created and presented throughout the world. Most scenarios are focused on learning how to recognize and treat rare, complex, and clinical problems. However, to our knowledge, this study is the first in which an HPS scenario has been focused on palpation with arrhythmias, basic but important skills. Arterial pulse waveforms, such as normal, water-hammer pulse, bifid pulse, and pulsus alternans, are usually monitored on a polygraph screen in practice. The volume of the pulse is a subtle sign to recognize low and high volume pulse (increased, normal or reduced) [1]. This medical skill requires years of experience with many patients and many careful examinations [1,2]. The pulsation and arrhythmias could not be realized even with standardized patients (actors and actresses trained to portray patients with specific clinical symptoms and conditions). Although this study mainly intended to deepen the participants' knowledge and interest in cardiovascular physiology, not to increase their clinical skills, they will assess pulsation exactly on real patients in the clinical setting.

The contemporary HPS has palpable pulses, heart, breath, and bowel sounds and the arm for intravenous infusion [4]. However, time was required for faculty to become sufficient familiar with the technology to be able to use it effectively for student learning $[6,8]$. We also recognized the mechanical limitations of the HPSs with

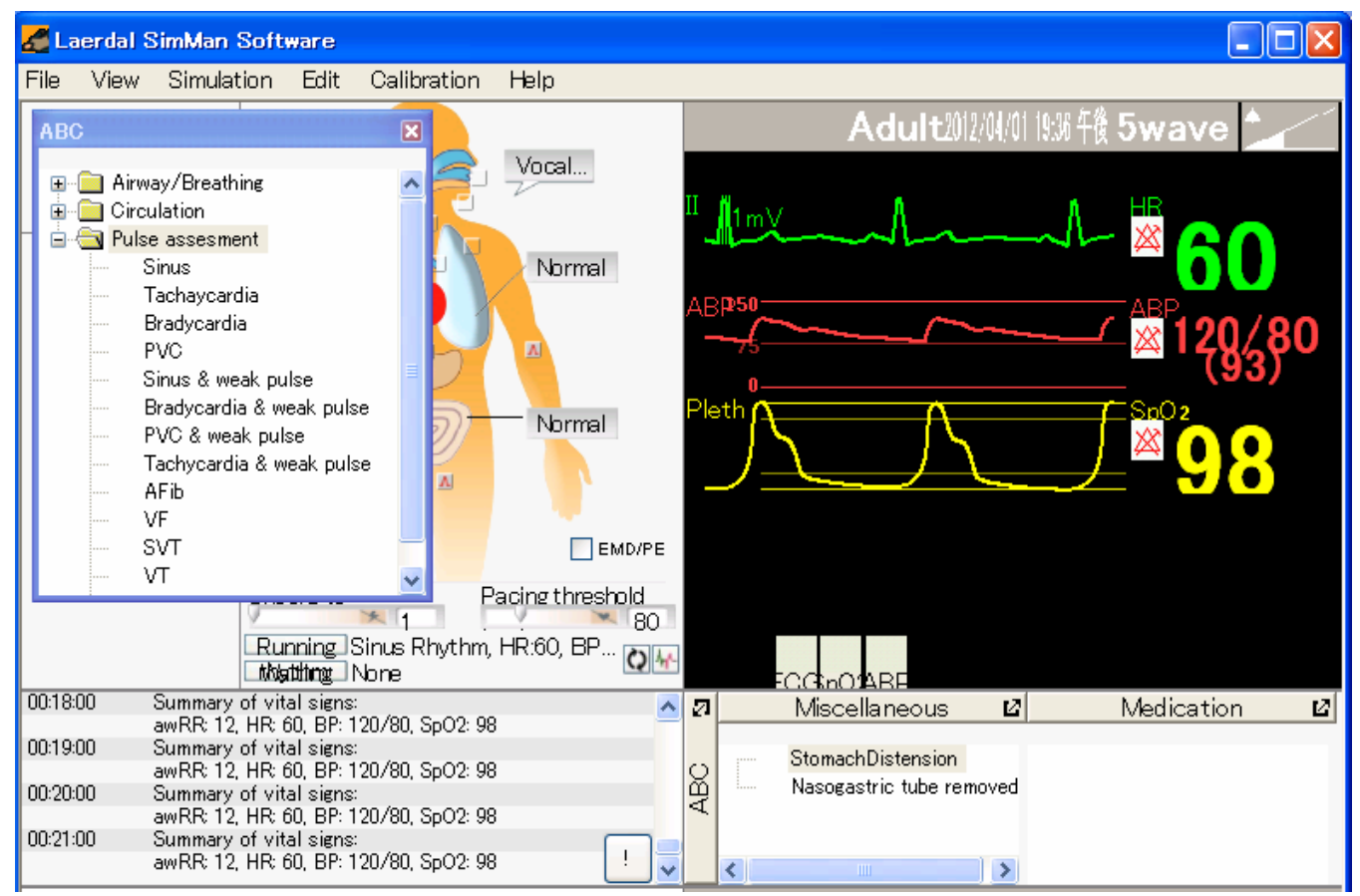

Figure 2. A control window on the main window of HPS. A list of training pulses is displayed in the control window. The ECG, arterial pulse and saturation of oxygen are sequentially drawn in a monitor panel on the main window. The two windows are only shown in operator's PC. The display is hidden to participants in training. 
Table 1. Summary of test scores and questionnaire results.

\begin{tabular}{|c|c|c|c|}
\hline Arrhythmia (pulse rate/min) & Pre-test & \multicolumn{2}{|c|}{ Post-test (corrected, $\mathrm{N}=25$ ) } \\
\hline Sinus (60) & $18(72 \%)$ & \multicolumn{2}{|c|}{$21(84 \%)$} \\
\hline Tachycardia (120) & $14(56 \%)$ & \multicolumn{2}{|c|}{$23(92 \%)$} \\
\hline Bradycardia (40) & $15(60 \%)$ & \multicolumn{2}{|c|}{$23(92 \%)$} \\
\hline Premature ventricular contraction (80) & $2(4 \%)$ & \multicolumn{2}{|c|}{$18(72 \%)$} \\
\hline Sinus (60 with weak pulse) & $2(4 \%)$ & \multicolumn{2}{|c|}{$11(44 \%)$} \\
\hline Bradycardia (40 with weak pulse) & $3(12 \%)$ & \multicolumn{2}{|c|}{$12(44 \%)$} \\
\hline PVC (80 with weak pulse) & $2(8 \%)$ & \multicolumn{2}{|c|}{$18(72 \%)$} \\
\hline Tachycardia (120 with weak pulse) & $4(16 \%)$ & \multicolumn{2}{|c|}{$25(100 \%)$} \\
\hline Atrial fibrillation (60) & $1(4 \%)$ & \multicolumn{2}{|c|}{$8(32 \%)$} \\
\hline Ventricular fibrillation (0) & $0(0 \%)$ & \multicolumn{2}{|c|}{$19(76 \%)$} \\
\hline Questionnaire for participants' knowledge about arrhythmias & \multicolumn{3}{|l|}{$(\mathrm{N}=25)$} \\
\hline Do you have any arrhythmias? & \multicolumn{2}{|c|}{ Yes (often, sometimes) 3} & No 22 \\
\hline Have you learned about arrhythmias in any lectures? & Yes $21 \quad \mathrm{I}$ & & \\
\hline \multicolumn{4}{|l|}{ How would you rate your understanding of arrhythmias? } \\
\hline Only names of some diagnoses 1 & e diagnoses 20 & Ability & ead an ECG 4 \\
\hline Have you measured your own pulse of the radial artery correctly? & Yes 12 & & No 13 \\
\hline Do you know there is a palpitation function on HPSs? & Yes 7 & & No 18 \\
\hline Questionnaire for participants' impressions of the training & & & \\
\hline How would you rate your understanding of pulses of the radial arte & & & \\
\hline Deepened $15 \quad$ Better $10 \quad$ Not improved 0 & fused 0 & & \\
\hline How well do you feel the training helped you understand arrhythm & & & \\
\hline $\begin{array}{llll}\text { Strongly } 21 & \text { Fairly } 3 & \text { Not well } 0 \text { Not effect }\end{array}$ & ive 0 & & \\
\hline How well do you feel the training helped you understand cardiovas & cular physiology & & \\
\hline $\begin{array}{lll}\text { Strongly } 11 & \text { Fairly } 13 \text { Not well } 1 \text { Not effect }\end{array}$ & ive 0 & & \\
\hline How would you recommend this training to a colleague? & & & \\
\hline Strongly $15 \quad$ Slightly 9 Not well 1 Not at all & & & \\
\hline How do you assess the training? (Comments) & & & \\
\hline . "Sensing pulses with my fingers is important for studying arrhyt & mias.” & & \\
\hline . "Sensing pulses deepens my understanding of medical knowledg & e with realism.” & & \\
\hline . “I received useful feedback from this training.” & & & \\
\hline . “This training was a good use of my time.” & & & \\
\hline . "This training should be a required component of medical educa & ion.” & & \\
\hline . “This training is relatively hard." & & & \\
\hline . "Increasing difficulty of the simulated pulses helps." & & & \\
\hline . "This training was fun.” Etc. & & & \\
\hline
\end{tabular}

making the scenarios. All mechanical parameters of the HPSs must completely be set in scenarios because the parameters are independent, not linked with other parameters, and constant. The used HPS can pulsate arteries as only normal, weak, or none, not perfectly replicate various pulse waveforms. In 1982, Kreitenberg et al. [1] created a simple teaching device for examination of the arterial and venous pulse that created palpable pulses with changing mechanical cams. The device exactly reproduced physiologic tracings of arterial pulsations, normal, bisferious, hyperkinetic, alternans, slow-rising, and anacrotic and dicrotic waves. For ECG, although the HPS simulates a fibrillated baseline of AF on a monitor screen, pulse volume on the artery is not changed without manual setting of arterial systolic and diastolic blood pressure in a scenario. RR intervals of AF are relatively constant, which may be misleading to understand real AF pulses. These results revealed inadequacies of current HPSs and suggest possible details and functions of the next generation of HPSs that would be more accurate and realistic. However, participants' evaluations revealed positive reviews of this palpation experience in HPS. 
Confucius said, "I hear and I forget. I see and I remember. I do and I understand.” Likewise, students' experience of palpation could indirectly deepen their understanding of arrhythmia and bridge the gaps between cardiovascular physiology and clinical phenomena.

\section{CONCLUSION}

We created an original palpation training scenario to detect pulses in the radial artery. The palpation training was evaluated with pre- and post-examination scores and positive comments. We also recognized the incompleteness of HPSs and that the details and functions of future HPSs can technically be improved.

\section{ACKNOWLEDGEMENTS}

We thank Robert E. Brandt (Founder, CEO, and CME, MedEd Japan, Suginami, Tokyo, Japan) for helpful advice on the English language in the preparation and editing of this manuscript.

\section{REFERENCES}

[1] Kreitenberg, A., Karliner, J.S., Engler, R.L. and Marchand, E.R. (1982) A simple teaching device for examination of the arterial and venous pulse. American Journal of Cardiology, 50, 1391-1393. doi:10.1016/0002-9149(82)90480-5
[2] Nair, B.K. (2011) The pulse. In: Nair, B.K., Ed. Adult Physical Examination. Accessed 22 August 2011. http://physicalexamination.org/?q=node/33

[3] Good, M.L. (2003) Patient simulation for training basic and advanced clinical skills. Medical Education, 37, 14-21. doi:10.1046/j.1365-2923.37.s1.6.x

[4] Wilson, M., Shepherd, I., Kelly, C. and Pitzner, J. (2005) Assessment of a low-fidelity human patient simulator for the acquisition of nursing skills. Nurse Education Today, 25, 56-67. doi:10.1016/j.nedt.2004.10.004

[5] Hyatt, J.P. and Hurst, S.A. (2010) Novel undergraduate physiology laboratory using a human patient simulator. Medical Education, 44, 523. doi:10.1111/j.1365-2923.2010.03651.x

[6] McCausland, L.L., Curran, C.C. and Cataldi, P. (2004) Use of a human simulator for undergraduate nurse education. International Journal of Nursing Education Scholarship, 1, 1-17. doi:10.2202/1548-923X.1035

[7] Wallin, C.J., Meurling, L., Hedman, L., Hedegård, J. and Felländer-Tsai, L. (2007) Target-focused medical emergency team training using a human patient simulator: Effects on behaviour and attitude. Medical Education, 41, 173-180. doi:10.1111/j.1365-2929.2006.02670.x

[8] Brannan, J.D., White, A. and Bezanson, J.L. (2008) Simulator effects on cognitive skills and confidence levels. Journal of Nursing Education, 47, 495-500. doi:10.3928/01484834-20081101-01 